\title{
SOME PROBLEMS OF ANALYTIC NUMBER THEORY - III
}

\author{
By R. BALASUBRAMANIAN AND K. RAMACHANDRA
}

\section{\& 1. Introduction}

The main theme of this paper is to systematize the Hardy-Landau $\Omega$ results and the Hardy $\Omega_{ \pm}$results [3] on the divisor problem and the circle problem. The method of ours is general enough to include tne abelian group problem and the results of Richert [9] and the later modifications by Warlimont [11], and in fact theorem 6 of ours is an improvement of their results. All our results are effective as in our earlier paper II [2] with the same title. Some of our results are new. For example $\Omega\left(x^{\frac{1}{6}}(\log x)^{\frac{1}{2}}\right)$ and $\Omega_{ \pm}\left(x^{a}\right)$, with $a=\frac{92}{1221}$, in the case of abelian groups. But our present methods for $\Omega_{ \pm}$results are imperfect in the sense that they depend on the known $O$-estimates for the error terms and so there is some loss and the exponents are not optimal. We first record our abelian group result as (for our earlier result see see [1] ).

\section{Theorem I}

Let $\mathrm{A}_{0}(x)$ denote the number of non-isomorphic abelian groups of orders not exceeding $x(x>10)$. An approximation to $\mathrm{A}_{0}(x)$ is $\sum_{j=1}^{20} \mathrm{C}_{j}{ }^{\frac{1}{j}}$ where $\mathrm{C}_{j} x^{\frac{1}{j}}$ is the residue at 
$s=\frac{1}{j}$ of $\left(\frac{x}{s}_{k=1}^{\infty} \zeta(k s)\right)$. We have $\mathrm{M}(\mathrm{I})>10^{-800}$ and $m(\mathrm{I})<-10^{-800}$, where $\mathrm{I}$ denotes the interval $\left(e^{v}, e^{1000 y}\right)$ and, $\mathrm{M}(\mathrm{I})$ and $m$ (I) denote respectively, the maximum and the minimum of the quantity

$$
\left(\mathrm{A}_{0}(x)-\sum_{j=1}^{20} \mathrm{C}_{j} x^{\frac{1}{j}}\right) x^{-a}(\log x)^{-\frac{1}{2}} \text { as } x^{-v a r i e s}
$$

over I. Here $a=\frac{92}{1221}$, and $y \geqslant 1000$. Also if $a=\frac{1}{6}$ the square of the last quantity has a maximum $\geqslant 10^{-8000}$ as $x$ varies over I.

Remark: Although we do not compute the constants explicitly our method surely leads to these constants. We get optimal results for the $\Omega$ problem in almost every case (Circle problem, Divisor problem and so on ) and we get for

$$
\left(\sum_{n \leqslant x} d(n)-(x \log x+(2 y-1) x)\right)^{2} x^{-\frac{1}{1}}
$$

and also for

$$
\begin{gathered}
\left\{\left(\begin{array}{c}
\Sigma \\
m^{2}+n^{2}<x \\
m, n+\text { ve, }- \text { ve or zero }
\end{array}\right)-\pi x\right\}^{2} x^{-\frac{2}{8}} \\
\end{gathered}
$$

results which are analogous to the statement of theorem 1. However for the analogue of the first statement, we have to take $a=\frac{1}{6}$ or slightly bigger exponents $(O$-results of G.A Kolesnik [4] enable us to take any $a<\frac{19}{108}$ ). The exponent 
$a=\frac{92}{1221}$ (in theorem 1 ) comes from the use of the $O$-estimate of B.R. Srinivasan (See Acta Arith., Vol. 23 (1973), 195-205) in our work. For an improvement of theorem 1 see $\$ 4$.

Our method of attack is as follows. Let $\mathrm{A}(x)$ be a complex valued (Riemann) integrable function defined by $\mathrm{A}(x)=0$ if $0<x<1$, and subject to $|\mathrm{A}(x)|<(x+2) \mathrm{C}$ in $x>1$ where $C$ is a positive constant. Then the following theorem is an easy consequence of a theorem, of $\mathrm{H}$. L Montgomery and R. C. Vaughan (for a simple proof of a special case which we actually need see [6].)

\section{Theorem 2}

$$
\text { Let } s=\sigma^{-}+\mathrm{it}, \sigma>0, \mathrm{~T} \leqslant t<2 \mathrm{~T}, \mathrm{X} \geqslant \mathrm{T}^{\frac{1}{2}},
$$

$\mathrm{Y} \geqslant \mathrm{T}^{3}+2 \mathrm{X}$ where $\mathrm{T} \geqslant 1000$. Then with

$$
\begin{aligned}
& 2+i \infty \\
& \Delta(x)=\frac{1}{2 \pi i} \int^{w} x^{w} \operatorname{Exp}\left(\mathrm{W}^{4 a+2}\right) \frac{d \mathrm{~W}}{\mathrm{~W}},(x>0) \\
& 2-i \infty \\
& =\frac{1}{\pi} \int_{0}^{\infty} \sin (v \log x) \exp \left(-v^{4 a+2}\right) \frac{d v}{v} \\
& (a \geq 0 \text { being an integer const int), we have, } \\
& \frac{1}{\mathrm{~T}} \int_{\mathrm{T}}^{2 \mathrm{~T}}\left|\int_{2 \mathrm{X}}^{\infty} u^{-s} \Delta\left(\frac{\mathrm{Y}}{u}\right) d(\mathrm{~A}(u))\right|^{2} d t \\
& =O\left(\left(\frac{\mathrm{T}}{\mathrm{X}}+\mathrm{T}\right) \int_{\mathrm{X}}^{\infty}\left|\frac{\mathrm{A}(u)}{\sigma}\right|^{2}\left(\Delta_{0}\left(\frac{\mathrm{Y}}{u}\right)\right)^{\mathrm{B}} \frac{d u}{u}\right),
\end{aligned}
$$

where $\mathrm{B}>0$ is an arbitrary constant, $\Delta_{0}(x)=\min (1, x)$, and the $O$-constant depends only on $a, \mathrm{~B}, \sigma$ and $\mathrm{C}$ (and in an effective way).

From this we deduce the following 


\section{Theorem 3}

Let $1=\lambda_{1}<\lambda_{2}<\ldots$ be a sequence of real numbers with $\frac{1}{\mathrm{C}}<\lambda_{n+1}-\lambda_{n}<\mathrm{C}$, where $\mathrm{C}$ is a constant $>1$, and $n=1,2,3, \ldots$ Let $\left\{a_{n}\right\}$ be a sequence of complex numbers subject to $\left|a_{n}\right| \leqslant(n+1)^{C}$. Let L be a curve consisting of finitely many fixed straight lines all contained in $(0 \leqslant \operatorname{Re} w \leqslant 1+\sigma,|\operatorname{Im} w|<C)$, where $\sigma>0$. Let $\mathrm{H}(\mathrm{W})$ be analytic function analytic on $\mathrm{L}$ such that $\frac{\mathrm{H}(\mathrm{W})}{\mathrm{W}}$ is analytic on $\mathrm{L}$ and let $\mathrm{L}$ be provided with a definite orientation. For $u>0$, put

$$
\mathrm{D}(u)=\frac{1}{2 \pi i} \int_{\mathrm{L}} \mathrm{H}(\mathrm{W}) u^{\mathrm{W}} \frac{d \mathrm{~W}}{\mathrm{~W}} .
$$

Then with $s=\sigma+$ it, $\sigma>0$ and

$\mathrm{A}(u)=\sum_{n}<u{ }_{n}-\mathrm{D}(u),(u>0)$,

$\mathrm{T}^{\frac{1}{2}}<\mathrm{X}<\mathrm{T}^{2}, \operatorname{Exp}\left(\mathrm{T}^{4 a}\right) \geqslant \mathrm{Y}>\mathrm{T}^{3}+2 \mathrm{X}$, we have,

$\frac{1}{\mathrm{~T}} \int_{\mathrm{T}}^{2 \mathrm{~T}} \mid \sum_{n=1}^{\infty} a_{n} \lambda_{n}^{-s} \Delta\left(\frac{\mathrm{Y}}{\lambda_{n}}\right)-\sum_{\lambda_{n}<2 \mathrm{X}} a_{n} \lambda_{n}^{-s} \Delta\left(\frac{\mathrm{Y}}{\lambda_{n}}\right)^{2} d t$ $=o\left(\frac{\mathrm{X}^{2}}{\mathrm{~T}^{2}}\right)+o\left(\left(\mathrm{~T}+\frac{\mathrm{T}}{\mathrm{X}}\right) \int_{\mathrm{X}}^{\infty}\left|\frac{\mathrm{A}(u)}{u^{\sigma}}\right|^{2}\left(\Delta_{0}\left(\frac{\mathrm{Y}}{u}\right)\right)^{\mathrm{B}} \frac{d u}{u}\right)$,

where $\mathrm{B}>0$ is any arbitrary constant, $\Delta_{0}(\mathrm{X})=\min (\mathrm{I}, \mathrm{X})$ $\mathrm{T} \geqslant 100$, and the $O$-constant depends only on a, $\mathrm{B}, \sigma$ and $\mathrm{C}$, it being assumed without loss of generality that $\max \left|\frac{\mathrm{H}(\mathrm{W})}{\mathrm{W}}\right|$ as $\mathrm{W}$ varies on $\mathrm{L}$ does not exceed $\mathrm{C}$. Moreover the O-constant is effective. 


\section{Remark}

The term $\frac{\mathrm{X}^{2}}{\mathrm{~T}^{2}}$ can be replaced by

$$
\frac{\mathrm{X}^{2}(\lambda-\sigma)}{\mathrm{T}^{2}}+\operatorname{Exp}\left(-\mathrm{T}^{4 a}\right) \mathrm{Y}^{2(\lambda-\sigma)},
$$

where $\lambda=\max \operatorname{Re}(w)$ as $w$ varies on $L$. It is completely absent if $\mathrm{D}(u) \equiv 0$ for $u>0$.

It is easy to indicate how theorem 3 follows from theorem 2 .

From $\mathrm{A}(u)=\sum_{\lambda_{n}<u} a_{n}-\mathrm{D}(u)$, it follows that

$\int_{2 \mathrm{X}}^{\infty} u^{-s} \Delta\left(\frac{\mathrm{Y}}{u}\right) d \mathrm{~A}(u)$

$=\sum_{\lambda_{n} \mathrm{X}} a_{n} \Delta\left(\frac{\mathrm{Y}}{\lambda_{n}}\right) \lambda_{n}^{-s}-\int_{2 \mathrm{X}}^{\infty} u^{-s} \Delta \frac{\mathrm{Y}}{\mathrm{D}_{n}(u) d u}$

$=\sum_{n=1}^{\infty} a_{n} \Delta\left(\frac{\mathrm{Y}}{\lambda_{n}}\right) \lambda_{n}^{-s}-\sum_{\lambda_{n} \leqslant 2 \mathrm{X}} a_{n} \Delta\left(\frac{\mathrm{Y}}{\lambda_{n}}\right)$

$$
\lambda_{n}^{-s}-I_{1}(s)+1_{2}(s) \text {, }
$$

where

$$
\begin{aligned}
l_{1}(s) & =\int_{0}^{\infty} u^{-s} \Delta\left(\frac{\mathrm{Y}}{u}\right)\left(\frac{1}{2 \pi i} \int_{\mathrm{L}} \mathrm{H}(\mathrm{W}) u^{\mathrm{W}-1} d \mathrm{~W}\right) d u \\
& =\frac{1}{2 \pi i} \int_{\mathrm{L}} \mathrm{H}(\mathrm{W}) \mathrm{J}_{1}(\mathrm{~W}-s) d \mathrm{~W} \quad \text { and }
\end{aligned}
$$




$$
\begin{aligned}
\mathrm{I}_{2}(s) & =\int_{0}^{2 \mathrm{X}} u^{-s} \Delta\left(\frac{\mathrm{Y}}{u}\right)\left(\frac{1}{2 \pi i} \int_{\mathrm{L}} \mathrm{H}(\mathrm{W}) u^{\mathrm{W}-1} d \mathrm{~W}\right) d u \\
& =\frac{1}{2 \pi i} \int_{\mathrm{L}} \mathrm{H}(\mathrm{W}) \mathrm{J}_{2}(\mathrm{~W}-s) d \mathrm{~W}
\end{aligned}
$$

where, for complex $Z$,

$$
\begin{aligned}
\mathbf{J}_{1}(\mathrm{Z}) & =\int_{0}^{\infty} u^{\mathrm{Z}-1} \Delta\left(\frac{\mathrm{Y}}{u}\right) d u \\
& =\mathrm{Y}^{\mathrm{Z}} \int_{0}^{\infty} v^{\mathrm{Z}-1} \Delta\left(\frac{1}{v}\right) d v \text { and } \\
\mathbf{J}_{2}(\mathrm{Z}) & =\int_{0}^{2 \mathrm{X}} u^{\mathrm{Z}-1}\left(1+\left(\Delta\left(\begin{array}{l}
\mathrm{Y} \\
u
\end{array}\right)-1\right)\right) d u . \\
& =\frac{(2 \mathrm{X})}{\mathrm{Z}}+\int_{0}^{\mathrm{Z}}\left(\Delta\left(\frac{\mathrm{Y}}{u}\right)-1\right) u^{\mathrm{Z}-1} d u .
\end{aligned}
$$

By using the result on page 7 (Chapter I, the section on Mellin transforms) of Titchmarsh's book [10], we find that $J_{1}(Z)=Y^{Z} Z^{-1} \operatorname{Exp}\left(Z^{4 a+2}\right)$. Also since $\Delta(x)=1$ $+O\left(x^{-B}\right)$ for every positive constant $B$, it follows that $J_{2}(Z)-(2 X)^{Z} Z^{-1}$ is an entire function of $Z$. Thus

$$
\begin{aligned}
& \int_{2 \mathrm{X}}^{\infty} u^{-s} \Delta\left(\frac{\mathrm{Y}}{u}\right) d \mathrm{~A}(u) \\
& =\sum_{n=1}^{\infty} a_{n} \Delta\left(\frac{\mathrm{Y}}{\lambda_{n}}\right) \lambda_{n}^{-s}-\sum_{\lambda_{n}<2 \mathrm{X}} a_{n} \Delta\left(\frac{\mathrm{Y}}{\lambda_{n}}\right) \lambda_{n}^{-s}
\end{aligned}
$$




$$
\begin{aligned}
& -\frac{1}{2 \pi i} \int_{\mathrm{L}} \mathrm{H}(\mathrm{W}) \mathrm{Y}^{\mathrm{W}-s^{\mathrm{s}}}(\mathrm{W}-s)^{-1} \leftarrow \\
& +\frac{1}{2 \pi i} \int_{\mathrm{Exp}\left((\mathrm{W}-s)^{4 a+2}\right) d \mathrm{~W}} \mathrm{H}(\mathrm{W})(2 \mathrm{X})^{\mathrm{W}-s} \frac{d \mathrm{~W}}{\mathrm{~W}-s} \\
& +\frac{1}{2 \pi i} \int_{\mathrm{L}} \mathrm{H}(\mathrm{W})\left(\int_{0}^{2 \mathrm{X}}\left(\Delta\left(\frac{\mathrm{Y}}{\mathrm{u}}\right)-1\right) u^{\mathrm{W}-s-1} d u\right) d \mathrm{~W}
\end{aligned}
$$

This is an identity valid for all complex $s$. Trivially this identity gives theorem 3 once theorem 2 is granted. We will prove theorem 2 in $\$ 2$.

Theorem 3 is a step in the proof of our main theorem which we prove in $\mid 3$, and is an interesting lemma by itself. Since for any two complex numbers $z_{1}, z_{2}$ we have $\left|z_{1}-z_{2}\right|^{2}>\frac{1}{2}\left|z_{1}\right|^{2}-\left|z_{2}\right|^{2}$, the LHS of the main inequality of theorem 3 is

$$
\begin{aligned}
& >\frac{1}{2 \mathrm{~T}} \int_{\mathrm{T}}^{2 \mathrm{~T}}|\mathrm{G}(s)|^{2} d t \\
& -\frac{1}{\mathrm{~T}} \int_{\mathrm{T}}^{2 \mathrm{~T}}\left|\sum_{\lambda_{n}<2 \mathrm{X}} a_{n} \lambda_{n}{ }^{-s} \Delta\left(\frac{\mathrm{X}}{\lambda_{n}}\right)\right|^{2} d t, \\
& \text { where } \mathrm{G}(s)=\sum_{n=1}^{\infty} a_{n} \lambda_{n}{ }^{-s} \Delta\left(\frac{\mathrm{Y}}{\lambda_{n}}\right),
\end{aligned}
$$

Here the second term (and in fact the mean value over $(0,2 \mathrm{~T}))$ is $\sum_{\lambda_{n}<2 \mathrm{X}}\left(1+o\left(\frac{\lambda_{n}}{\mathrm{~T}}\right)\right)\left|\frac{a_{n}}{\lambda_{n}}\right|^{2}$. 
where the $O$-constant can be taken to be $6 \pi$. Hence the main problem is to obtain fairly economical lower bounds for $2 \mathrm{~T}$

the mean value $\frac{1}{\mathrm{~T}} \int_{\mathrm{T}}|\mathrm{G}(\sigma+i t)|^{2} d t$. Then we choose $X$ properly and get lower bounds for

$$
\int_{\mathrm{X}}^{\infty}\left|\frac{\mathrm{A}(u)}{u^{\sigma}}\right|^{2}\left(\Delta_{0}\left(\frac{\mathrm{Y}}{u}\right)\right)^{\mathrm{B}} \frac{\mathrm{du}}{u} .
$$

If we now substitute an upper bound for: $\mathbf{A}(u) \mid$ then we end up with a lower bound for

$$
\int_{\mathrm{X}}^{\infty}\left|\frac{\mathrm{A}(u)}{u^{2 \sigma-l}}\right|\left(\Delta_{0}\left(\frac{\mathrm{Y}}{u}\right)\right)^{\mathrm{B}} \frac{d u}{u}
$$

where $l$ is a non-negative constant coming from the loss due to a (possibly) non-optimal upper bound for |A $(u) \mid$.

While the first lower bound leads to an $\Omega$ result which is nearly optimal the second leads to $\Omega_{ \pm}$resuits which are not optimal by the method of our earlier paper [2]. In both these results it is necessary to see that $\mathrm{Y}$ does not exceed a fixed power of $\mathrm{T}$.

The main problem which we concentrate in this paper is the case $\lambda_{n}=n, \mathrm{~F}(s)=\sum_{n=1}^{\infty} a_{n} n^{-s}$ being the $k^{t h}$ power of a Dirichlet series $F_{0}(s)=\sum_{n=1}^{\infty} b_{n} n^{-s}$. Here $k$ is a positive integer constant $\geqslant 2$. All that we need is that $F_{0}$ converges somewhere in the complex plane and that it should admit an analytic continuation in $(\sigma>t-8$, T $<t<2 \mathrm{~T})$ for some positive constant and in that region 
$\max \mathrm{F}_{0}(s) \mid<\operatorname{Exp}\left(\mathrm{T}^{\mathrm{C}}\right), \mathrm{T}>100$. We have to borrow a result from Ramachandra's paper [8]. Using this we deduce from Theorem 3 an $\Omega$ result which is nearly optimal. We prefer to state the General theorem on $F(s)=\left(\mathrm{F}_{0}(s)\right)^{k}$ in $\S 3$. All our results are effective. Next we would like to say that it is possible to unify the circle problem and the divisor problem and state a general theorem on $F(s)=F_{0}(s) F_{1}(s)$, where $\mathrm{F}_{0}(s)$ has a functional equation and $\mathrm{F}_{1}(s)$ is a Dirichlet series satisfy ing some conditions. We can also generalize, to some extent, the result

$$
\frac{1}{\mathrm{~T}} \int_{\mathrm{T}}^{2 \mathrm{~T}}\left|\prod_{k=1}^{\infty} \tau\left(k\left(\frac{1}{6}+\mathrm{it}\right)\right)\right|^{2} d t \gg \mathrm{T} \log \mathrm{T},
$$

whose proof will be sketched after the statement of theorem 4 .

Before closing the introduction we record here a theorem on the zeros of Dirichlet series which can be obtained by the method of an earlier paper, (On the zeros of a class of generalised Dirichlet series V, J. fur. Reine u. Angew. Math, $303 / 304$ (1978), 295-313) by the second of us. The reason is that the function $\Delta(x)$ employed in the present paper is very helpful and can be used to improve Theorem 16 of that paper. However we state only a special case of the improvement for simplicity. The full paper with details will appear as a joint paper by us as a continuation of Ramachandra's paper $V$ cited above, as paper VI with the same title.

\section{Theorem 4}

Let $\left\{a_{n}^{\prime}\right\}$ be a sequence of complex numbers such that ${ }_{n}^{\prime}=O$ (1) and $\frac{1}{x} \sum_{x<n<2 \text { : }} \operatorname{Re}\left(a_{n}\right)$ is bounded below for all $x$ exceeding a certoin posirive constant. Suppose that the Dirichlet 
series $\sum_{n=1}^{\infty} a_{n}^{\prime} n^{-s}$ can be continued in $\left(\cdot>\frac{1}{2}-\delta\right.$, $\mathrm{T}<\mathrm{t}<2 \mathrm{~T}$ ) analytically and the absolute value of the function does not exceed a fired power of $\mathrm{T}$. Then the number of zeros of the function in $\left(\sigma>\frac{1}{2}-\delta, \mathrm{T}<t \leqslant 2 \mathrm{~T}\right)$ is $>\mathrm{T}(\log \mathrm{T})^{1-\varepsilon}$ provided $\mathrm{T}$ is large enough and $0<\varepsilon<\frac{1}{10}, 0<\delta<\frac{1}{10}$. The constant which $\mathrm{T}$ should erced is effective.

To close up the introduction we sketch a proof that

$$
\left.\left.\frac{1}{\mathrm{~T}} \int_{\mathrm{T}}^{2 \mathrm{~T}}\right|_{k} ^{\infty} \pi \zeta(k s)\right|^{2} d t \gg \mathrm{T} \log \mathrm{T} \text { on } \sigma=\frac{1}{6} .
$$

By the functional equation this result follows from a similar result where the integrand is replaced by

$$
\begin{aligned}
& \mathrm{T}\left|{\underset{k=1}{\pi}}_{k=4}^{3} \zeta(1-k s)\left(\pi_{k=4}^{6} \zeta(k s)\right)\right|^{2} \\
& \left.=\mathrm{T} \mid\left(\sum_{k=1}^{3} \zeta(1-2 k \sigma+k s)\right){ }_{k=4}^{(\pi} \zeta(k s)\right)\left.\right|^{2} \text {. }
\end{aligned}
$$

Now there are several methods of deducing the lower bound $\gg \mathrm{T} \log \mathrm{T}$. The most general and powerful method is to apply the following theorem.

\section{Theorem 5}

$$
\text { Let } \mathrm{F}(s)=1+\sum_{n=2}^{\infty} \frac{a^{\prime \prime}}{n^{s}} \text { be analytie in }\left(\cdot>\frac{1}{2}\right. \text {, }
$$

$\mathrm{T}<t<2 \mathrm{~T})$ and there $\max |\mathrm{F}(s)|<\operatorname{Exp}\left(\mathrm{T}^{\mathrm{A}}\right)$ where $\mathrm{A}$ is a posisive eonst int an $l \mathrm{~T}>10$. (The series for $\mathrm{F}(\mathrm{s})$ is assumed to 
SOME PROBLEMS OF ANALYTIC NUMBER THEORY-III

23

converge for at least one complex number s and the $a_{n}{ }^{\prime} s$ are assumed to be complex numbers with $\left.\left|a_{n}{ }^{\prime \prime}\right| \leq(n+1)^{\mathrm{A}}\right)$. Then

$$
\begin{aligned}
& \frac{1}{\mathrm{~T}} \int_{\mathrm{T}}^{2 \mathrm{~T}}\left|\mathrm{~F}\left(\frac{1}{2}+\mathrm{it}\right)\right|^{2} d t \gg \\
& \quad \sum_{n} \mid \frac{\mathrm{T}}{100}+1
\end{aligned}
$$

where the constant implied by the Vinogradov symbol $\gg$ depends only on $\mathrm{A}$.

Remark

This is the special case of a more general theorem of Ramachandra (See [7].)

Thus the result

$$
\frac{1}{\mathrm{~T}} \int_{\mathrm{T}}^{2 \mathrm{~T}}\left|\sum_{k=1}^{\infty} \pi(k s)\right|^{2} d t \gg \mathrm{T} \log \mathrm{T},\left(\cdot=\frac{1}{6}\right)
$$

is completely proved except for the verification that for the

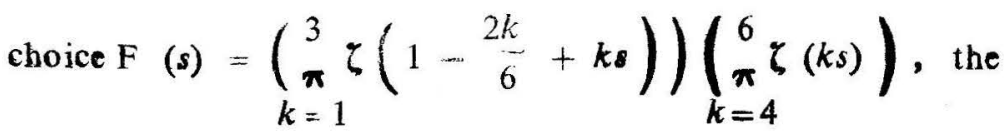
right hand side of the main inequality of theorem 5 is $\gg \log T$. This is left as an easy exercise.

82. Proof of Theorems 2 and 3.

We begin with

Lemma I. Subject to the convergence of integrals and subject to $\mathrm{Y} \geqslant 2 \mathrm{X}$, we have

$$
\int_{\mathrm{X}}^{2 \mathrm{X}}\left\{\frac{\mathrm{I}}{\mathrm{T}} \int_{\mathrm{T}}^{2 \mathrm{~T}}\left|\int_{\mathrm{X}}^{\infty} u^{-s} \Delta\left(\frac{\mathrm{Y}}{u}\right) d \mathrm{~A}(u)\right|^{2} d t\right\} \frac{d x}{x} .
$$




$$
=o\left(\left(\sigma^{2}+\mathrm{T}^{2}\right)\left(\frac{1}{\mathrm{X}}+\frac{1}{\mathrm{~T}}\right) \mathrm{E}_{1}\right),
$$

where, $\mathrm{X}>2, \mathrm{~T}>2, s=\sigma+\mathrm{it}$, and,

$$
\mathrm{E}_{1}=\int_{\mathrm{X}}^{\infty}\left|\frac{\mathrm{A}(u)}{u^{\sigma}}\right|^{2}\left(\min \left(1, \frac{\mathrm{Y}}{u}\right)\right) \text { B } \frac{d u}{u}
$$

where $\mathrm{B}>0$ is any constant, and the 0 -constant depends only on $\mathrm{B}$ and $a$.

Proof.

We apply Montgomery - Vaughan theorem (see [6]) as follows. Putting $f(u)=u^{-s} \Delta\left(\frac{Y}{u}\right)$, we see that

$$
\begin{aligned}
\text { LHS }= & \int_{\mathrm{X}}^{2 \mathrm{X}}\left\{\frac{1}{\mathrm{~T}} \int_{\mathrm{T}}^{2 \mathrm{~T}} \mid[\mathrm{A}(u) f(u)]_{u=x}^{\infty}\right. \\
& \left.-\left.\int_{x}^{\infty} \mathrm{A}(u) f^{\prime}(u) d u\right|^{2} d t\right\} \frac{d x}{x}
\end{aligned}
$$

$$
\begin{aligned}
& <2 \int_{\mathrm{X}}^{2 \mathrm{X}}\left|\frac{\mathrm{A}(x)}{X^{\sigma}}\right|^{2}\left(\Delta\left(\frac{Y}{x}\right)\right)^{2} \frac{d x}{x} \\
& \quad+2 \int_{\mathrm{X}}^{2 \mathrm{X}}\left\{\frac{1}{\mathrm{~T}} \int_{\mathrm{T}}^{2 \mathrm{~T}}\left|\int_{\mathrm{X}}^{\infty} \mathrm{A}(u) f^{\prime}(u) d u\right|^{2} d t\right\} \frac{d x}{x} .
\end{aligned}
$$

Next we observe that the innermost integral in the second term here

$$
=\int_{0}^{1}\left(\sum_{n=0}^{\infty}\left(\mathrm{A}(n+u+x) f^{\prime}(n+u+x)\right)\right) d u
$$

and so the second term does not exceed 


$$
\begin{aligned}
& 4 \int_{\mathrm{X}}^{2 \mathrm{X}}\left\{\int_{0}^{1}\left(\sum_{n=0}^{\infty}\left|\frac{\mathrm{A}(n+u+x)}{(n+u+x)^{\sigma}}\right|^{2}{ }_{(n+u+x)}^{2}\left(\frac{\sigma^{2}+4 \mathrm{~T}^{2}}{(n+u+2}\right)\right)^{2}\right. \\
& \left.\left.\left.+\frac{\mathrm{Y}^{2}}{(n+u+x)^{4}}\left(\Delta^{\prime}\left(\frac{\mathrm{Y}}{n+u+x}\right)\right)^{2}\right) \mathrm{E}_{2}(n+u+x)\right) d u\right\} \frac{d x}{x}
\end{aligned}
$$

where $E_{2}=1+O\left(\frac{n+u+x}{T}\right)$ (and the $O$-constant can be taken to be $6 \pi$ since $\log \left(1+\frac{1}{n}\right) \geqslant \frac{1}{2 n}$ for $\left.n>1\right)$.

Hence the second term does not exceed

$$
4 \int_{\mathrm{X}}^{2 \mathrm{X}}\left\{\int_{\mathrm{X}}^{\infty}\left|\frac{\mathrm{A}(u)}{u^{\sigma}}\right|^{2} \mathrm{E}_{3}(u) \frac{d u}{u}\right\} \frac{d x}{x}
$$

where $\mathrm{E}_{3}(u)=\left(\frac{\sigma^{2}+4 \mathrm{~T}^{2}}{\mathrm{~m}}\left(\Delta\left(\frac{\mathrm{Y}}{u}\right)\right)^{2}+\right.$

$$
\left.\frac{\mathrm{Y}^{2}}{u^{3}}\left(\Delta^{\prime}\left(\frac{\mathrm{Y}}{u}\right)\right)^{2}\right)\left(1+o\left(\frac{u}{\mathrm{~T}}\right)\right) \text {. }
$$

Now

$$
\begin{aligned}
\mathrm{E}_{3}(u) & =\frac{\sigma^{2}+4 \mathrm{~T}^{2}}{u}\left(\Delta\left(\frac{\mathrm{Y}}{u}\right)\right)^{2}+\frac{\mathrm{Y}^{2}}{u^{3}}\left(\Delta^{\prime}\left(\frac{\mathrm{Y}}{u}\right)\right)^{2} \\
& +o\left(\frac{\sigma^{2}+4 \mathrm{~T}^{2}}{\mathrm{~T}}\left(د\left(\frac{\mathrm{Y}}{u}\right)\right)^{2}+\frac{\mathrm{Y}^{2}}{u^{2} \mathrm{~T}}\left(\Delta^{\prime}\left(\frac{\mathrm{Y}}{u}\right)\right)^{2}\right) .
\end{aligned}
$$

Next for $u>\mathrm{Y}$ we have $\Delta\left(\frac{\mathrm{Y}}{u}\right)=O\left(\left(\frac{\mathrm{Y}}{u}\right)^{\mathrm{B}}\right)$,

$$
\Delta^{\prime}\left(\frac{\mathrm{Y}}{u}\right)=O\left(\left(\frac{\mathrm{Y}}{u}\right)^{\mathrm{B}}\right)=O\left(\left(\frac{\mathrm{Y}}{u}\right)^{-\mathrm{B}}\right)
$$


for every $\mathrm{B}>0$ and hence the constribution from $u>\mathrm{Y}$ is

$$
\begin{aligned}
& o\left(\int _ { \mathrm { X } } ^ { 2 \mathrm { X } } \left\{\int _ { \mathrm { Y } } | \frac { \mathrm { A } ( u ) } { \sigma } | _ { u } ^ { 2 } \left(\frac{\sigma^{2}+4 \mathrm{~T}^{2}}{u}-\left(\frac{\mathrm{Y}}{u}\right)^{\mathrm{B}}+\frac{\mathrm{Y}^{2}}{u^{3}}\left(\frac{\mathrm{Y}}{u}\right)^{\mathrm{B}-2}\right.\right.\right. \\
& \left.\left.\left.+\frac{\sigma^{2}+4 \mathrm{~T}^{2}}{\mathrm{~T}}\left(\frac{\mathrm{Y}}{u}\right)^{\mathrm{B}}+\frac{\mathrm{Y}^{2}}{u^{2} \mathrm{~T}}\left(\frac{\mathrm{Y}}{u}\right)^{\mathrm{B}-2}\right) \frac{d u}{u}\right\} \frac{d x}{x}\right)
\end{aligned}
$$

where $B$ is any positive constant.

For $u<\mathrm{Y}$ the contribution is [by using $\Delta^{\prime}(\mathrm{X})=O\left(\mathrm{X}^{\mathrm{B}}\right)$ $=O\left(\mathrm{X}^{-\mathrm{B}}\right)$ and $\Delta(\mathrm{X})=1+O\left(\mathrm{X}^{-\mathrm{B}}\right)$, for every constant B $>$ 0]

$$
\begin{array}{r}
o\left(\int _ { \mathrm { X } } ^ { 2 \mathrm { X } } \left\{\int _ { \mathrm { X } } | \frac { \mathrm { A } ( u ) } { u ^ { \sigma } } | ^ { 2 } \left(\frac{\sigma^{2}+4 \mathrm{~T}^{2}}{u}+\frac{1}{u}\right.\right.\right. \\
\left.\left.\left.\quad+\frac{\sigma^{2}+4 \mathrm{~T}^{2}}{\mathrm{~T}}+\frac{1}{\mathrm{~T}}\right) \frac{d u}{u}\right\} \frac{d x}{x}\right) \\
=O\left(\int _ { \mathrm { X } } ^ { 2 \mathrm { X } } \left\{\int _ { \mathrm { X } } | \frac { \mathrm { Y } ( u ) } { u ^ { \sigma } } | ^ { 2 } \cdot \left(\frac{\sigma^{2}+4 \mathrm{~T}^{2}}{u}+\frac{1}{u}\right.\right.\right. \\
\left.\left.\left.+\frac{\sigma^{2}+4 \mathrm{~T}^{2}}{\mathrm{~T}}+\frac{1}{\mathrm{~T}}\right)\left(\min \left(1, \frac{\mathrm{Y}}{u}\right)\right)^{\mathrm{B}} \frac{d u}{u}\right\} \frac{d x}{x}\right)
\end{array}
$$

and hence the LHS of Lemma 1 is

$$
\begin{array}{r}
o\left(\int _ { \mathrm { X } } \int _ { \mathrm { X } } \left\{\int_{u^{\sigma}}^{\infty}\left|\frac{\mathrm{A}(\mathrm{u})}{u^{2}}\right|^{2}\left(\frac{\sigma^{2}+4 \mathrm{~T}^{2}}{u}+\frac{\sigma^{2}+4 \mathrm{~T}^{2}}{\mathrm{~T}}\right)\right.\right. \\
\left.\left.\left(\min \left(1, \frac{\mathrm{Y}}{u}\right)\right)^{\mathrm{B}} \frac{d u}{u}\right\} \frac{d x}{x}\right) .
\end{array}
$$

This gives the lemma.

Lemma I easily gives theorem 2 and th in turn gives theorem 3 in a fairly obvious way as was already seen in the introduction. 


\section{3. The Main Theorem.}

We now start with an easy lemma. As stated already we put

$$
\begin{aligned}
& \mathrm{F}_{0}(s)=\sum_{n}^{\infty} \frac{b_{n}}{n^{s},} \\
& \left(\mathrm{~F}_{o}(s)\right)^{k}=\mathrm{F}(s)=\sum_{n=1}^{\infty} \frac{a_{n}}{n^{s}}, \alpha+\frac{1}{2}-\frac{1}{2 k} .
\end{aligned}
$$

Lemma 2. For all $\mathrm{N}>1$, we have,

$$
\begin{aligned}
& \sum_{1 \leqslant n<\mathrm{N}}\left|a_{n} n^{-\alpha}\right|^{2}= \\
& o\left(\mathrm{~N}^{\frac{1}{k}+\varepsilon}\left(\sum_{1<n \leqslant \mathrm{~N}}\left|b_{n} n^{-\frac{1}{2}}\right|^{2}\right)^{k}\right),
\end{aligned}
$$

where $\varepsilon>0$ is arbitrary and the $O$-constant depends only on $\varepsilon$ and $k$.

Proof:

$$
\begin{aligned}
& \text { LHS }=\sum\left|b_{n_{1}} \ldots b_{n_{k}} b_{n_{1}} \ldots b_{n_{k}},\left(n_{1} \ldots n_{k}\right)^{-2 \alpha}\right| \\
& n_{1} n_{2} \ldots n_{k}=n_{1}^{\prime} n_{2}^{\prime} \ldots n_{k}^{\prime}=n<\mathrm{N} \\
& \leqslant 2 \sum\left|b_{n_{1}} \ldots b_{n_{k}}\right|^{2} d\left(n_{1} \ldots n_{k}\right)\left(n_{1} \ldots n_{k}\right)^{-2 \alpha} \\
& n_{1} \ldots n_{k} \leqslant \mathrm{~N} \\
& =O\left(\mathrm{~N}^{\boldsymbol{\varepsilon}} \sum\left|b_{n_{1}} \ldots b_{n_{k}}\right|^{2}\left(n_{1} \ldots n_{k}\right)^{-2 \alpha}\right) \\
& n_{1} \ldots n_{k} \leqslant \mathrm{~N}
\end{aligned}
$$


The lemma follows on applying a simple inductive argument on $k$, to estimate the last sum. Here we have used

$$
\left|\mathrm{z}_{1} \mathrm{z}_{2}\right|<\left|\mathrm{z}_{1}\right|^{2}+\left|\mathrm{z}_{2}\right|^{2}
$$

We next state our main theorem and sketch its proof.

\section{Theorem 6.}

$$
\text { Let } \mathrm{F}_{o}(s)=\sum_{n=1}^{\infty} \frac{b_{n}}{n^{s}} \text { be a Dirichlet series with complex }
$$

coefficients $b_{n}$, which converges at least at one point of the complex plane and define $\mathrm{F}(s)=\left(\mathrm{F}_{o}(s)\right)^{k}$ where the series for $\mathrm{F}_{0}(s)$ is absolutely convergent (note shat if $\mathrm{F}_{o}(s)$ converges at $s$ it converges absolutely at $s+2)$. Let $\alpha=\frac{1}{2}-\frac{1}{2 k}$ and let

A be a constant $\geqslant 2 k$. Suppose that in $\left(\sigma>\frac{1}{2}-\frac{1}{\mathrm{~A}}, \mathrm{~T}\right.$

$$
1-\frac{1}{A}
$$

$<t \leqslant 2 \mathrm{~T}), \mathrm{F}_{0}(s)$ admits an analytic continuation and there $\mathrm{M}$ defined by $\mathrm{M}=\max \left|\mathrm{F}_{o}(s)\right|$ coes not exceed $\operatorname{Exp}\left(\mathrm{T}^{\mathrm{B}}\right)$ where $\mathrm{B}$ is a positive constant, and $\mathrm{T} \geqslant 10$. Let $\mathrm{L}$ be a curve consisting of finitely many fixed strcight lines all contsined in $(0 \leqslant \mathrm{Re}$ $w<1+\alpha$, $|\operatorname{Im} w|<\mathrm{B}$ ), and assume that $\mathrm{H}$ (W) is an analytic function (defined on $\mathrm{L}$ ) such that $\frac{\mathrm{H}(\mathrm{W})}{\mathrm{W}}$ is analytic on L. Put

$$
\mathrm{D}(u)=\frac{1}{2 \pi i} \int_{\mathrm{L}} \mathrm{H}(\mathrm{W}) u^{\mathrm{W}} \frac{d \mathrm{~W}}{\mathrm{~W}}, \quad(u>0)
$$

and next

$$
\mathrm{E}(u)=\sum_{n \leqslant u} a_{n}-\mathrm{D}(u), \quad(u>0) .
$$


Let $\alpha_{1}, \alpha_{2}, \alpha_{3}, \theta$ an $1 \mathrm{C}$ be positive constants subject to $\alpha<\frac{1}{2}-\frac{1}{\mathrm{~A}}<\alpha_{1}<\alpha_{2}<\alpha_{3}<\frac{1}{2}, \theta<1-\frac{1}{k}$ and further assume thit the maximum of $\left|b_{n}\right|$ for $1<n<$ $(100)^{-1} \mathrm{~T}^{1-\frac{1}{\mathrm{~A}}}$ exceeds $\mathrm{T}^{-\mathrm{C}}$ and $\left|{ }_{n}\right|^{1}<(n+1)^{\mathrm{C}}$ $(n=1,2,3, \ldots$,$) and \max _{\mathrm{W} \text { on } \mathrm{L}}\left|\frac{\mathrm{H}(\mathrm{W})}{\mathrm{W}}\right|<\mathrm{C}$. Then there exists an effective positive constant $\mathrm{T}_{0}=\mathrm{T}_{0}\left(k, \mathrm{~A}, \mathrm{~B}, \mathrm{C}, \boldsymbol{\theta}, \alpha_{1}\right.$, $\left.\alpha_{2}, \alpha_{3}\right)>2$ such that for all $\mathrm{T}>\mathrm{T}_{0}$ there holds,

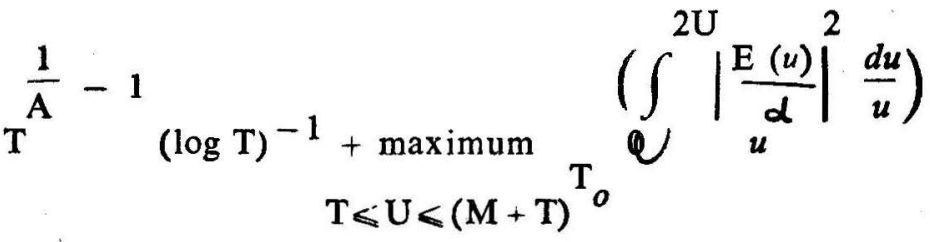

$>\mathrm{T}_{0}^{-1}$ minimum

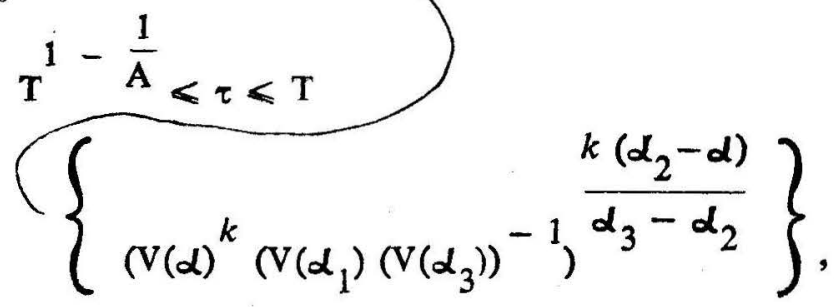

where $\mathrm{V}(\sigma)=\frac{1}{\tau} \quad \sum\left|b_{n}\right|^{2}\left(\frac{\tau}{n}\right)^{2 \sigma}$, provided only

$$
n \leqslant \frac{\tau}{100}
$$

that $\left(\sum_{n<\mathrm{T}} n^{-1}\left|b_{n}\right|^{2}\right)^{k}$ does not exceed $\mathrm{T}^{0}$ times the

RHS of the main inequality. 
Remark. Actually the term $\mathrm{T}^{\frac{1}{\mathrm{~A}}}-1$ (log $\left.\mathrm{T}\right)^{-1}$ on the LHS can be replaced by $(\log T)^{-1} T^{-(3-2 \lambda+2 \alpha)\left(1-\frac{1}{A}\right)}$

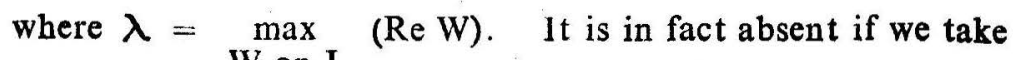
W on $\mathrm{L}$ $H(W)=0$.

Corollary. We have $\left(\right.$ with $\left.(\zeta(s))^{k}=\sum_{n=1}^{\infty} \frac{d_{k}(n)}{n^{s}}\right)$, $\sum_{n<\mathrm{X}} d_{k}(n)=\mathrm{D}_{k}(\mathrm{X})+\Omega\left(\mathrm{X}^{\alpha}\right)$, where $\alpha=\frac{1}{2}-\frac{1}{2 k}$ and $\mathrm{D}_{k}(\mathrm{X}) \mathrm{X}^{-1}$ is a polynomial in $\log \mathrm{X}$ of degree $k-1$. In particular $\mathrm{D}_{2}(\mathrm{X}) \mathrm{X}^{-1}$ is $\log \mathrm{X}+2 \mathrm{\gamma}-1$, where $\gamma$ is the Euler's constant.

Our method gives $\Omega\left(\mathrm{X}^{\frac{5}{4}}\right)$ for the circle problem as well. We can formalise in this direction to some extent but not yet with such a full satisfaction as we have given Theorem 6 .

In the rest of this section we sketch the remaining part of the proof of theorem 6 . We will first handle the function

$$
G(s)=\sum_{n=1}^{\infty}\left(a_{n} n^{-s} \Delta\left(\frac{Y}{n}\right)\right)
$$

where $\mathbf{Y}=(\mathrm{M}+\mathrm{T})^{b}$ where $b>10$ is a positive constant depending on $A$ and $\alpha_{1}$. Let $H=T^{\frac{1}{b}}$. Now $G(s)$ is an entire function and it is very close to $F(s)$ in 


$$
\left(\sigma>\frac{1}{2}\left(\frac{1}{2}-\frac{1}{\mathrm{~A}}+\alpha_{1}\right), \mathrm{T}^{1-\frac{1}{\mathrm{~A}}}+\mathrm{H} \leqslant t<2 \mathrm{~T}-\mathrm{H}\right) .
$$

Also for $\mathrm{X}=\mathrm{T}, \mathrm{T} \leqslant t \leqslant 2 \mathrm{~T}$ and $\sigma>0$, we have,

$\int_{2 \mathrm{X}}^{\infty} u^{-s} \Delta\left(\frac{\mathrm{Y}}{u}\right) d(\mathrm{E}(u)+\mathrm{D}(u))-\mathrm{G}(s)+O\left(\mathrm{X}^{(3 \mathrm{C}+10) k}\right)$.

Thus on integrating by parts, we get,

$\int_{2 \mathrm{X}}^{\infty} \frac{d}{d u}\left(u^{-s} \Delta\left(\frac{\mathrm{Y}}{u}\right)\right) \mathrm{E}(u) d u$

$=-\int_{2 \mathrm{X}}^{\infty} u^{-s} \Delta\left(\frac{\mathrm{Y}}{u}\right) \mathrm{D}^{\prime}(u) d u+\mathrm{G}(s)+O\left(\mathrm{~T}^{(6 \mathrm{C}+20) k}\right)$

$=\mathrm{G}(s)+O\left(\mathrm{~T}^{(6 \mathrm{C}+20) k}\right)$,

by an argument similar to the one we used, in the introduction, to deduce theorem 3 from theorem 2. In other words if $|\mathrm{G}(s)|$ exceeds a large constant power of $\mathrm{T}$ somewhere in

$$
\begin{aligned}
& 1-\frac{1}{A} \\
& (\sigma>0, T<t<2 \mathrm{~T}) \text { then } \\
& \int_{2 \mathrm{X}}^{\infty}\left|\frac{d}{d u}\left(u^{-s} \Delta\left(\frac{\mathrm{Y}}{u}\right)\right) \mathrm{E}(u)\right| d u
\end{aligned}
$$

(and hence $\int_{\mathrm{X}}^{\infty} \ldots d u$ ) also exceeds a large constant power of $\mathbf{T}$ for this value of $s$. From this it is not hard to show that our Theorem 6 follows except when $|\mathrm{G}(s)|$ is bounded by a constant power of $\mathrm{T}$ in $\left(\sigma>0, \mathrm{~T}^{1-\frac{1}{\mathrm{~A}}}<t<2 \mathrm{~T}\right)$. We 
now consider the excluded case which is possibly exceptional. As already remarked $F(s)$ is close to $G(s)$ in

$$
\left(\sigma>\frac{1}{2}\left(\frac{1}{2}-\frac{1}{\mathrm{~A}}+\alpha_{1}\right), \mathrm{T}^{\mathrm{I}-\frac{1}{\mathrm{~A}}}+\mathrm{H} \leqslant t \leqslant 2 \mathrm{~T} \quad \mathrm{H}\right)
$$

Hence in this region $|F(s)|$ is bounded by a constant power of $\mathrm{T}$. Hence we can take a new $\Delta$ transform $\mathrm{G}_{1}(s)$ of $\mathrm{F}(s)$ (with a different $Y$, now a large constant power of $T$ ) in place of $\mathrm{G}(s)$, such that in

$$
\left(\sigma>\frac{1}{4}\left(\frac{1}{2}-\frac{1}{\mathrm{~A}}+3 \alpha_{1}\right), \mathrm{T}^{1-\frac{1}{\mathrm{~A}}}+\mathrm{H}<t<2 \mathrm{~T}-\mathrm{H}\right)
$$

$\mathrm{G}_{1}(s)$ is a good approximation to $\mathrm{F}(v)$. It follows now from the results of Ramachandra's paper [8] (see Theorem 4 of [8]) that

$$
\frac{\int_{T}^{1-\frac{1}{A}}+2 H}{2 T-2 H}\left|G_{1}(\alpha+i t)\right|^{2} \frac{d t}{t^{2}}
$$

$\gg \Psi \log \mathrm{T} \quad$ minimum

$$
\left.\left(\begin{array}{l}
T^{1-\frac{1}{A}}<t \leqslant 2 T
\end{array}\right) \frac{k\left(\alpha_{2}-\alpha\right)}{\alpha_{3}-\alpha_{2}}\right\}
$$

where the constant implied by the Vinogradov's symbol is effective. Theorem 3 and lemma 2 now complete the proof of theorem 6. We have only to replace $A$ by a larger constant which is not a loss of generality.

mearem 3 reeds a shipat morificalion is insters $\frac{1}{T} \int_{T}^{2 T} 11^{2} d t$ we have $t$ consiler $\int_{T^{1-}}^{2 T} 11^{2} \frac{d t}{t_{2}}$. mis in a rimelle was sias 
8. Some Concluding Remarks. In our remarks preceeding theorem 4 we have stated that it is possible to unify the circle lattice point problem, the divisor problem and generalise it very :much. We can also generalise the abelian group problem very much. We can proceed much along the lines of this paper of course with an appropriate generalisation of the results of [7] and [8]. But our $\Omega_{ \pm}$results are not so satisfactory as our results [2] which are linked up with the singularities. Our $\boldsymbol{\Omega}$ results are fairly satisfactory but our $\Omega_{ \pm}$results are not. For example the abelian group result reads $\Omega_{ \pm}\left(\mathrm{X}^{a}\right)$ with $a=\frac{92}{1221}$ and the expected result is $\Omega_{ \pm}\left(\mathrm{x}^{\frac{1}{6}}\right)$. This imperfection results from the fact that we have to depend upon the known $O$-results which are far from being satisfactory.

However there is one method of quite a general character which leads to

$$
\underset{n \leq \mathrm{X}}{\Sigma} d_{k}(n)=\mathrm{D}_{k}(\mathrm{X})+\Omega_{ \pm}\left(\mathrm{X}^{\frac{1}{2}-\frac{1}{\mathrm{R}}}(\log \mathrm{X})^{-1}\right)
$$

$(k$, an integer constant $>3)$ and

$$
\Omega_{ \pm}\left(X^{\frac{1}{10}} \operatorname{Exp}\left(\frac{3}{4}\left(\frac{\log X}{\log \log X}\right)^{\frac{1}{2}}\right)\right)
$$

in the abelian group problem. Moreover the results are effective in the same sense as: theorem 1 . For this we have some2X

how to get a lower bound for $\frac{1}{\mathrm{X}} \int_{\mathrm{X}}|\mathrm{E}(x)| d x$. The rest of the procedure is the same as in [2]. To obtain a lower bound for this first power mean value we prove that the generating functions $\mathbf{F}(s)\left[=(\zeta(s))^{k}\right.$ for the divisor problems and 
$=\prod_{k=1}^{\infty} \zeta(s k)$ for the abeiian group problem] have a large absolute value fairly frequently on the relevant lines $\omega=\frac{1}{2}-\frac{1}{k}$ for the divisor problem, $\cdot=\frac{1}{10}$ for the abelian group problem). This leads to the results. We now state two theorems.

\section{Theorem 7 .}

Let $k>3$ and let $b_{n}$ be all real and let the conditions of theorem be satisfied with $\alpha=\frac{1}{2}-\frac{1}{k}$ (instead of $\left.\alpha=\frac{1}{2}-\frac{1}{2 k}\right)$ and $\mathrm{A}>k$ (instead of $\mathrm{A} \geqslant 2 \mathrm{k}$ ). Then for all $\mathrm{T} \geqslant \mathrm{T}_{o}=$ $\mathbf{T}_{0}\left(k, \mathrm{~A}, \mathrm{~B}, \mathrm{C}, 0, \alpha_{1}, \alpha_{2}, \alpha_{3}\right), \quad\left(\mathrm{T}_{0} \geqslant 2\right)$ there holds

$$
\begin{aligned}
& \frac{1}{\mathrm{~T}}+(\log \mathrm{T}) \quad \max \quad \frac{\mathrm{E}(u)}{u^{\alpha}} \\
& \mathrm{T}^{\frac{1}{\mathrm{~T}}} \leqslant \mathrm{U} \leqslant(\mathrm{M}+\mathrm{T})^{\mathrm{T}} \text { o } \\
& >\mathrm{T}_{0}^{-1}(\mathrm{~V}(\alpha))^{\frac{k}{2}}\left(\mathrm{~V}\left(\alpha_{1}\right)\left(\mathrm{V}\left(\alpha_{3}\right)\right)^{-1}\right)^{\frac{k\left(\alpha_{2}-\alpha\right)}{2\left(\alpha_{3}-\alpha_{2}\right)}}
\end{aligned}
$$

where $\mathrm{V}(\sigma)$ is as before, (but with $\tau=\mathrm{T}$ ) provided that $\left(\sum_{n<\mathrm{T}} n^{-1}\left|b_{n}\right|^{2}\right)^{k / 2}$ does not exceed $\mathrm{T}^{\theta}$ times the RHS of the main inequality. Also the same ineruality holds with $-\frac{\mathrm{E}(u)}{u^{\alpha}}$ in place of the quantity $\frac{\mathrm{E}(u)}{u^{\alpha}}$. Further we have to make the assumptions that $\mathrm{F}(s)=\left(\mathrm{F}_{o}(s)\right)^{k}$ is regular (at $s=\frac{1}{2}-\frac{12}{11 k}$ and) in $\sigma \geqslant \frac{1}{2}-\frac{12}{11 k}$ except possibly for singularities on the 
line $t=0$ and that $\mathrm{H}(\mathrm{W})=\mathrm{F}(\mathrm{W})$ and further $\mathrm{L}$ is an antclockwise oriented rectangle enclosing all the singularities of $\mathrm{F}(\mathrm{s})$ which lie in $\left(\alpha-\frac{1}{11 k}<\sigma \leqslant 1+\alpha, t=0\right)$ and $\sigma>1+\alpha$ is free from the singularities of $\mathrm{F}(s)$. Finally without compltcating the other conditions we have to impose that

RHS $>\operatorname{Exp}\left(-\frac{\log \mathrm{T}}{20 k}\right)$.

Corollary :

$$
\sum_{n<x} d_{k}(n)=\mathrm{D}_{k}(x)+\Omega_{ \pm}\left(x^{\frac{1}{2}-\frac{1}{k}}(\log x)^{-1}\right),
$$

for $k>3$ and $\mathrm{D}_{k}(x)=\frac{1}{2 \pi i} \quad \int_{|s-1|=(\zeta(s))^{k} x^{s} \frac{d s}{s}}$ $|s-1|=\frac{1}{10}$

We next state one more theorem.

\section{Theorem 8.}

Let $\mathrm{M}^{*}$ (I) and $m^{*}$ (1) denote the maximum and minimum of the quantity (for notation not explained here, see Theorem I)

$$
\left(\mathrm{A}_{0}(x)-\sum_{j=1}^{20} \mathrm{C}_{j} x^{\frac{1}{j}}\right) x^{-\frac{1}{10}} \operatorname{Exp}\left(-\frac{3}{4}\left(\frac{\log x}{\log \log x}\right)^{\frac{1}{2}}\right)
$$

in the interval I defined as $\left(e^{y}, e^{100 y}\right), y>1000$.

Then, we have, $\mathrm{M}^{*}(\mathrm{I})>10^{-800}$ and $m^{*}(\mathrm{I})<-10^{-800}$.

We now make some remarks about the proofs of theorem 7. The first step in proving the two theorems is Theorem 9.

We have, with the notation of theorem 7 ,

$$
\frac{1}{T}+(\log \mathrm{T}) \max _{\mathrm{T}<U<(M+\mathrm{T}) \mathrm{T}_{o}} \int_{U}^{2 U}\left|\frac{\mathrm{E}(x)}{\alpha}\right| \frac{d x}{x}
$$




$$
>\mathrm{T}_{0}^{-1}(\mathrm{~V}(\alpha))^{k / 2}\left(\mathrm{~V}\left(\alpha_{1}\right)\left(\mathrm{V}\left(\alpha_{3}\right)\right)^{-1}\right)^{\frac{k\left(\alpha_{2}-\alpha\right)}{2\left(\alpha_{3}-\alpha_{2}\right)}}
$$

Proof: Follows from the fact that we can assume first of all that $\mid G(s)$ | does not exces $d$ a fixed power of $T$. Next we derive a lower bound for

$$
\left|\int_{2 \mathrm{X}}^{\infty} \frac{\mathrm{E}(u)}{u^{s+1}} d u\right|(s=\alpha+\mathrm{it})
$$

from the mean square (over ( $T, 2 T$ )) lower bound for it. From this theorem 9 follows.

We now observe that if $f(u)=\left(\mathrm{E}\left(e^{u}\right)\right) e^{-u \alpha}$, then

$$
\int_{U}^{2 U}\left|\frac{E(x)}{x^{\alpha}}\right| \frac{d x}{x}<\int_{\log U-1 \log 2-\lambda}^{\log U+\frac{1}{2} \log 2+\lambda}|f(u)| d u,
$$

$(\lambda>0)$. We now put $\lambda=u_{1}+u_{2}+\ldots+u_{m}$ and integrate over a cube of dimension $m$ of side length $\wedge$ (a positive constant, which is small).

Denoting the average over the cube by $A(A, U, m)$, we have,

$$
\begin{gathered}
\frac{1}{\mathrm{~T}}+(\log \mathrm{T}) \quad \max \mathrm{T}_{\mathrm{T}} \mathrm{A}(\Lambda, \mathrm{U}, m) \\
>\mathrm{RHS} \text { of Theorem } 9 .
\end{gathered}
$$

Next we denote the average above but this time with $|f(u)|$ replaced by $f(u)$ by $\mathrm{B}(\Lambda . \mathrm{U}, m)$. We see that $\mathrm{B}(\Lambda, U, m)$ is the average of

$$
\begin{aligned}
& \frac{1}{2}(f(u+0)+f(u-0)) \\
& =\int_{\substack{\mathrm{C}_{1}-i \infty \\
|\mathrm{I} m \mathrm{~W}|>g}}^{\mathrm{C}_{1}+i \infty} \mathrm{F}(\mathrm{W}) e^{u(\mathrm{~W}-\alpha)} \frac{d \mathrm{~W}}{2 \pi i \mathrm{~W}}
\end{aligned}
$$




$$
\begin{aligned}
& +\int_{\alpha-\delta-i g}^{\alpha-\delta+t g} \mathrm{~F}(\mathrm{~W}) e^{u(\mathrm{~W}-\alpha)} \frac{d \mathrm{~W}}{2 \pi i \mathrm{~W}} \\
& +2 \text { other integrals over horizontal paths } \\
& =\mathrm{I}_{1}+\mathrm{I}_{2}+\mathrm{I}_{3}+\mathrm{I}_{4} \text { say, }
\end{aligned}
$$

where $g$ is a positive constant and $\delta$ is equal to $\frac{1}{11 k} ; C_{1}>0$ is a suitable constant. The average of $I_{2}$ is $O\left(e^{-u_{0} \delta}\right)$, where $u_{o}$ is the smallest value of $u$ in process of averaging. The other integrals have in all the average $O\left((2 \Lambda)^{m} e^{u_{1} C_{1}} g^{-m}\right)$ where $u_{1}$ is the largest value of $u$ in the process of averaging and so $u_{1}<\log U+m \wedge$. Put $m=\left[10^{-8} \log U\right]$ and $\Lambda=\frac{1}{10}$ Choose $g$ to be a large constant. This shows that $B(\Lambda, U, m)$ $=O\left(e^{-u_{0} \delta}\right)=O\left(u^{-\delta / 2}\right)$. To deduce Theorem 7 we need Theorem 10.

If $Q>0$ and if in the cube occurring in the definition of $\mathrm{A}(\Lambda, \mathrm{U}, m)$ and $\mathrm{B}(\Lambda, \mathrm{U}, m)$ we denote by $\mathrm{J}(\mathrm{Q})$ the portion where $|f(u)|>Q$ then either $f(u)$ changes sign in the region $J(Q)$ or

$$
\mathrm{A}(\Lambda, \mathrm{U}, m)<2 \mathrm{Q}+|\mathrm{B}(\Lambda, \mathrm{U}, m)| \text {. }
$$

From these remarks theorem 7 follows.

In the case of the abelian group problem we have to start with the following result

Theorem II.

$$
\text { We have, with } \sigma=\frac{1}{10} \text { and } s=\sigma+i t \text {, }
$$

$$
\max _{\mathrm{T}<t<2 \mathrm{~T}}\left(||_{k=1}^{\infty} \zeta(k s) \mid\right)>\mathrm{T} \operatorname{Exp}\left(\frac{3}{4}\left(\frac{\log \mathrm{T}}{\log \log \mathrm{T}}\right)^{\frac{1}{3}}\right),
$$

for all $\mathrm{T}$ exceeding a positive constant. 


\section{Remark :}

This is due to us and extends over some papers on "Titchmarsh's phenomenon". This is nearly the best known result in this direction. See a paper by $R$. Balasubramanian (to appear) to whom the constant $\dot{s}$ and in fact a better constant is due. From theorem 11 it follows as before that

$$
\int_{T}^{T}\left|\frac{E(u)}{u^{\sigma}}\right| \frac{d u}{u}>\operatorname{Exp}\left(\frac{3}{4}\left(\frac{\log T}{\log \log T}\right){ }^{3}\right)
$$

From this result theorem 8 follows very much like theorem 7 .

We hope to publish a fuller account of our investigations on $\Omega \pm$ results when they reach some finality.

\section{References}

1. R. Balasubramanian and K. Ramachandra, Effective and non-effective results on certain arithmetical functlons, J. of Number theory, 12 (1980), 10-19.

2. R. Balasubramanian and K. Ramachandra, Some problems of analytic number theory-II, (to appear).

3. G. H. Hardy, On Dirichlet's divisor problem, Proc. London Math. Soc. 2. (15) (1916), 1-25.

4. G. A. Kolesnik, On the order of $\zeta\left(\frac{1}{2}+i t\right)$ and $\Delta(R)$, (to appear).

5. E. Landau, (For references to the earlier papers of G. H. Hordy and those of Landau see [3]).

6. K. Ramachandra, Some remarks on a theorem of Montgomery and Vaughan, J. of Number theory, 11 (1975), 465-471.

7. K. Ramachandra, Progress towards a conjecture on the mean-value of Titchmarsh series, Proceedings of the Durham Conference on analytie number theory, (JulyAugust 1979) (to appear). 
8. K. Ramachandra, Progress lowards a conjecture on the mean-value of Titchmarsh series-1I, Hardy-Ramanujan Journal 4 (1981). 1-12.

9. H. E. Richert, Zur Multiplicativen Zahlentheorie, J. fur Reine u. Angew. Math, 206 (1961), 31-38.

10. E. C. Titchmarsh, Introduction to the theory of Fourier integrals, Oxford (1967).

11. R. Warlimont, Uber einen Satz von H. E. Richert Zur Multiplicativen Zahlentheorie, J. fur. Reine $u$. Angew Math. 221 (1966), 197-202.

Note: There are a few references which are important but which have not been included above for some reasons. They are

(A) B. R. Srinivasan, On the number of Abelian groups of a given order, Acta Arithmetica. Vol. 23 (1973), $195-205$.

(B) R. Balasubramanian, On the frequency of Titchmarsh's phenomenon for $\zeta(s)-\mathrm{IV}$, (to appear). For our earlier work in connection with Titchmarsh's phenomenon see this paper of $\mathbf{R}$. Balasubramanian.

(C) W. Schwarz, Uber die Anzahl Abelscher gruppen gegebner ordnung II, J. fur Reine $u$. Angew. Math, 228 (1967), $133-138$.

(D) P. G. Schmidt, Error term in the abelian group problem $o_{\varepsilon}\left(w^{10 / 39+\varepsilon}\right)$, Vortragsbuch Nr 31, Oberwolfach (1975), Seile 271 .

In theorem 8, we can take at present, only the expression.

$$
\begin{array}{r}
\left(A_{o}(x)-\sum_{j=1}^{20} \mathrm{C}_{j} x^{\frac{1}{j}}\right) x^{-\frac{1}{10}} \\
\operatorname{Exp}\left(-\frac{1}{20}\left(\frac{\log x}{\log \log x}\right)^{\frac{1}{2}}\right) .
\end{array}
$$


But we have not changed theorem 8 for some reasons. Indeed we hope to prove a stronger version with things like $x^{-\frac{1}{6}}$ in place of $x^{-\frac{1}{10}}$

Finally we note for comparison the result

$\int_{\mathrm{X}}^{2 \mathrm{X}}\left|\left(\mathrm{A}_{0}(x)-\sum_{j=1}^{20} \mathrm{C}_{j} x^{\frac{1}{j}}\right) x^{-\frac{1}{6}}\right|^{2} d x=O\left(\mathrm{X}(\log \mathrm{X})^{2}\right)$,

which can be proved by standard methods.

Manuscript completed On 11 Jan 1980. We noticed some improvements and hence revised on 25 Nov. 1980.

School of Mathematics

Tata Institute of Fundamental Research

Homi Bhabha Road

Bombay 400005

India. 